\title{
Effects of abdominal drawing-in maneuver with pressure biofeedback, foam-roller and quadruped on lumbopelvic stability and muscle activities in lumbar rotation syndrome
}

\author{
Dong-Hyun Kim, Tae-Ho Kim* \\ Department of Physical Therapy, College of Rehabilitation Science, Daegu University, Daegu, Korea
}

The abdominal drawing-in maneuver (ADIM) exercise has been commonly applied to recover lumbopelvic stability and normal movement in the clinical field. Although many researchers investigated its effects using several methods, there is further need for comparing those methods. The purpose of this study was to assess the effects of ADIM with three different methods including a pressure biofeedback unit, a foam-roller, and the quadruped posture on lumbopelvic rotation and muscle activities. Twenty-five healthy participants (14 males, 11 females) who volunteered for this study were randomly divided into three intervention groups. We obtained pre- and postintervention data through application of a three-dimensional motion analysis system and a surface electromyography. Results showed that lumbopelvic rotation degree was significantly decreased in all groups $(P<0.05)$. The muscle activities of external oblique, internal oblique of left side, and gluteus maximus were significantly increased $(P<0.05)$. The muscle activities of erector spinae and biceps femoris were not significantly different $(P>0.05)$. The muscle activity of internal oblique of the right side showed significant differences $(P<0.05)$, between ADIM with pressure biofeedback group and ADIM with foam-roller group, and ADIM with foam-roller group and ADIM with quadruped group. Three kinds of ADIM were effective to recover lumbopelvic stability. This study suggests that the proper method of ADIM should be applied to a given patient considering abilities to maintain equilibrium and to control muscular strength.

Keywords: Lumbar rotation syndrome, Lumbopelvic rotation, Lumbopelvic stability, Abdominal drawing-in maneuver

\section{INTRODUCTION}

One of the most common musculoskeletal issues is low back pain. A functional dysfunction caused by structural issues necessarily affects muscle contraction and equilibrium during movement. In the context of this concept, Sahrmann named it "movement impairment syndrome," and demonstrated that it is caused by stimuli from tissues of fascia and joint (Sahrmann et al., 2017). On the basis of the movement impairment syndrome, a researcher tested lumbopelvic movements watching upper and lower limbs because movement of a limb transmits load to the lumbopelvic region (Weyrauch et al., 2015).
Lack of lumbopelvic stability often causes the compensations, such as lumbopelvic flexion-extension and rotation during movements (Sahrmann et al., 2017). When a subject who has insufficient ability to control abdominal muscles performed this test actively, lumbopelvic compensations were clearly shown (Scholtes et al., 2009). Therefore, it is important to improve the regulatory ability of abdominal muscles to prevent compensations. To do so, abdominal drawing-in maneuver (ADIM) exercise, which is useful to activate abdominal deep muscles, has been applied commonly. Several previous studies reported that ADIM reduced lumbopelvic compensations during side-lying hip external rotation test and prone knee extension test (Cynn et al., 2006; Oh et al., 2007).
${ }^{*}$ Corresponding author: Tae-Ho Kim (D) https://orcid.org/0000-0001-7939-2139 Department of Physical Therapy, College of Rehabilitation Science, Daegu University, 201 Daegudae-ro, Jillyang-eup, Gyeongsan 38453, Korea E-mail: ptkimth@daegu.ac.kr

Received: January 18, 2019 / Accepted: March 3, 2019
This is an Open Access article distributed under the terms of the Creative Commons Attribution Non-Commercial License (http://creativecommons.org/licenses/by-nc/4.0/) which permits unrestricted non-commercial use, distribution, and reproduction in any medium, provided the original work is properly cited. 
Many researchers have reported the effects of ADIM and other stabilizing exercises. In various positions and postures with various procedures, ADIM and other stabilizing exercises have produced good effects and have been applied in clinical fields. A study suggested the merits of using pressure biofeedback for lumbopelvic stability and reported abdominal contraction exercise with pressure biofeedback improved contractile force of internal oblique and led to improvement of transverse abdominis (Lee et al., 2013).

Many previous studies have used the foam-roller, which is a kind of unstable surface, to achieve trunk stability. The studies demonstrated that foam-roller exercises found the great improvement of all the abdominal muscles activities (Kim et al., 2011; Kim et al., 2013b). A study in which subjects exercised prone knee extension on foam-roller confirmed good efficiency of the exercise for trunk stability and improved posterior oblique sling (Kim et al., 2013a).

Various tasks on the quadruped position, for instance, single arm extension, single leg extension, and bird-dog posture, contributed stability, cooperation, and tenderness to the movement. A study demonstrated good effects of those exercises (Pirouzi et al., 2013). For these reasons, ADIM exercise will be applied to three different kinds of interventions to analyze lumbopelvic motion and muscle activities in the present study.

Many studies have examined effectiveness of exercises for lumbopelvic stability. However, few previous studies compared more than two exercise types; none has compared the effects of ADIM exercises among pressure biofeedback, foam-roller and quadruped types. Therefore, this study aimed to compare the effects of ADIM exercise with the three different methods (pressure biofeedback, foam-roller, and quadruped posture) and to suggest proper uses for each. In addition, this study was a cross-sectional study on the basis of a previous study that reported that the first step of exercise improved muscle activity and reduced recurrence rate of the abnormal symptom as well as the abnormal symptom itself (Hall et al., 2009).

\section{MATERIALS AND METHODS}

\section{Subjects}

Twenty-five healthy adults (14 males, 11 females) participated in this study. All participants were provided written informed consent, and the study was approved by the Daegu University Institutional Review Board (1040621-201611-HR-012-02).

Subjects were randomly divided into three intervention groups:
Table 1. General characteristics of subjects $(n=25)$

\begin{tabular}{lccrc}
\hline \multirow{2}{*}{ Characteristic } & \multicolumn{3}{c}{ LRS } & P-value \\
\cline { 2 - 4 } & APB $(\mathrm{n}=8)$ & AFR $(\mathrm{n}=9)$ & AQP $(\mathrm{n}=8)$ & \\
\hline Age $(\mathrm{yr})$ & $27.38 \pm 3.88$ & $22.78 \pm 4.02$ & $21.75 \pm 3.24$ & 0.02 \\
Height $(\mathrm{cm})$ & $166.63 \pm 7.32$ & $168.67 \pm 8.74$ & $169.25 \pm 9.48$ & 0.94 \\
Weight $(\mathrm{kg})$ & $63.62 \pm 12.38$ & $63.22 \pm 10.52$ & $66.25 \pm 7.55$ & 0.86
\end{tabular}

Values are presented as mean \pm standard deviation.

LRS, lumbar rotation syndrome; $A P B$, abdominal drawing-in maneuver (ADIM) with pressure biofeedback group; AFR, ADIM with foam-roller group; AQP, ADIM with quadruped group.

ADIM with pressure biofeedback group (APB), ADIM with foam-roller group (AFR), and ADIM with quadruped group (AQP). Their descriptive demographic information is in Table 1. Exclusion criteria included the history of spinal pain, neurologic conditions, or musculoskeletal conditions that could interfere with single hip extension in the testing position. Leg dominance was determined by asking the subjects to kick a ball and the kicking leg was selected as the dominant leg (Van Deun et al., 2007). All participants were right-handed and dextropedal.

\section{Three-dimensional motion analysis system recording and data processing}

To measure the lumbopelvic rotation degree before and after the interventions, a three-dimensional motion analysis system (CMS 70P, Zebris Medizintechnik, Gmbh, Isny, Germany) was used. Two sets of triple markers for the measurement of spine motions were applied with elastic Velcro strips. The system components were the CMS 70P basic unit with data transmission, the MA-XX measurement unit with three ultrasound transmitters, cable adapter, ultrasound body triple markers, and computer system. The Windata 1.71 software was used for data analysis.

A set of triple marker for measurement of motions of the pelvis was placed on the posterior superior iliac spine to measure lumbopelvic motion: lumbopelvic flexion-extension (Pfe), lumbopelvic lateral bending $(\mathrm{Plb})$, and lumbopelvic rotation (Pro). Another triple marker was aligned vertically to the midline of the trunk to measure thoracic motion: angles of thoracic flexion-extension (Tfe), thoracic lateral bending (Tlb), and thoracic rotation (Tro). The measuring sensor was located at a distance of $1 \mathrm{~m}$ from the subject. The sampling rate for this study was $30 \mathrm{~Hz}$ and used six markers, six angles, and $80^{\circ}$ of inclination. The unit of motion was degree $\left({ }^{\circ}\right)$.

\section{Surface electromyography recording and data processing}

To measure the muscle activities of external oblique, internal 
oblique, erector spinae, gluteus maximus, and biceps femoris before and after the interventions, wireless surface electromyography (EMG; TeleMyo DTS, Noraxon Inc., Scottsdale, AZ, USA) was used. The TeleMyo DTS directly transmits myoelectric data from the electrode to a belt-worn receiver.

The points of the EMG electrodes were determined in accordance with previous studies (Hall et al., 2009; Lee et al., 2013; Matthijs et al., 2014). Disposable $\mathrm{Ag} / \mathrm{AgCl}$ surface electrodes were attached $2 \mathrm{~cm}$ apart to each muscle.

Electrodes for external oblique were placed below the rib cage and along a line connecting the inferior point of the costal margin and the contralateral pubic tubercle. For internal oblique, electrodes were placed immediately medially to the anterior superior iliac spine. For erector spinae, electrodes were placed $5 \mathrm{~cm}$ laterally from spinous processes of L4. For gluteus maximus, electrodes were placed at the middle point between sacral vertebrae and greater trochanter and the same level of greater trochanter. For biceps femoris, electrodes were placed $2 \mathrm{~cm}$ apart, parallel to the muscle fibers on the lateral aspect.

The maximal voluntary isometric contraction (MVIC) was measured for the target muscles to normalize EMG activity. Each subject was asked to voluntarily and maximally contract each muscle for 5 sec against the manual resistance of the examiner. The MVIC value used the average root mean square of three trials. The average EMG activity was expressed as a percentage of the MVIC value ( $\%$ MVIC).

Before attaching EMG electrodes, all sites for the attaching point were shaved with razors and deterged with an alcohol-soaked paper towel. The sampling rate was $1,000 \mathrm{~Hz}$ and EMG signals were filtered at $40-400 \mathrm{~Hz}$ by finite impulse response bandpass and $60-\mathrm{Hz}$ filters and infinite impulse response rejector and the rectification.

\section{Experimental procedures}

The APB group lay on the flat floor placing one hand on their left side and holding a pressure gauge of the pressure biofeedback unit with another hand. And subjects bent their legs with soles of the feet on the floor. An inflatable inelastic bag of the pressure biofeedback unit was placed under L4-5, and the scale of the pressure gauge was set at $40 \mathrm{mmHg}$. First, subjects slowly pulled their lower abdomen and navel toward back to contract transvers abdominis and set up the scale of the pressure gauge at $50 \mathrm{mmHg}$. Second, they lifted one foot and did a single knee extension and hip extension. After reaching full range of knee extension and when a line connecting anterior superior iliac spine to patella ran parallel to the floor, subjects stopped hip extension and maintained the posture for $5 \mathrm{sec}$. Third, they returned to the starting position. This intervention was tried on the basis of a previous study in which they administered the pressure biofeedback exercise to contract transvers abdominis independently (Lee et al., 2013).

The AFR group was permitted to rest temporarily on the floor with their fingertips. Because subjects should lie on a foam-roller, which is an unstable surface, they needed to become familiar with it to keep balance (Kim et al., 2011). Subjects lay on the foamroller placing their arms on their chest and bent their legs with soles of the feet on the floor. First, they slowly pulled their lower abdomen and navel toward the back. Second, subjects lifted one foot and did a single knee extension and hip extension. After reaching full range of knee extension and when a line connecting anterior superior iliac spine to patella ran parallel to the floor, subjects stopped hip extension and maintained the posture for $5 \mathrm{sec}$. Third, they returned to the starting position. Keeping transvers abdominis contraction and breathing during intervention term, the subjects repeated single leg flexion-extension.

The AQP group assumed quadruped posture, and subjects maintained the posture during the whole intervention term (Pirouzi et al., 2013). First, subjects slowly pulled their lower abdomen and navel toward the back to contract transvers abdominis. Second, they did a single shoulder flexion and contralateral hip extension, which is named the bird-dog posture. When a line connecting the lifted arm and leg ran parallel to the floor, they stopped lifting arm and leg and maintained the posture for five seconds. Third, they returned to the starting position.

All groups repeated the single leg flexion-extension and tried to keep contracting transvers abdominis and breathing during interventions. They repeated each exercise on both sides of legs; intervention period was ten seconds, and after 1-min repetition they took a rest for thirty seconds. Twenty times of flexion-extension totally were performed for $30 \mathrm{~min}$.

For pretest and posttest, subjects performed right hip extension in the quadruped posture. Before the tests, the investigator applied triple markers of three-dimensional motion analysis system and EMG electrodes on the appointed points of the subjects. The subjects performed right hip extension without knee flexion until their back of ankle reached to a fixed set point, and maintained that posture for $5 \mathrm{sec}$. Then, they returned to the starting position. They repeated these 3 times.

\section{Statistical analyses}

Wilcoxon Signed-Rank test was used to analyze the changes of 
lumbopelvic rotation degree and muscle activity in each group. And Kruskal-Wallis test was used to analyze the changes of lumbopelvic rotation degree and muscle activity among the three groups. A value of $P<0.05$ was considered to indicate statistical significance. Mann-Whitney test was used for post boc analysis. Statistical analyses were performed using SPSS ver. 18.0 (SPSS Inc., Chicago, IL, USA).

\section{RESULTS}

In $\mathrm{APB}$, there were significant increases in Tfe, Tlb, Pfe, and Pro $(P<0.05)$, but not a significant difference in Tro and $\mathrm{Plb}(P>$ $0.05)$. There were significant increases in external oblique, internal oblique and gluteus maximus $(P<0.05)$, but not a significant difference in erector spinae and biceps femoris $(P>0.05)$ (Table 2 ).

In AFR, there were significant increases in Tlb, Tro, $\mathrm{Pfe} \mathrm{Plb}$ and Pro $(P<0.05)$, but not a significant difference in Tfe $(P>$ $0.05)$. There were significant increases in external oblique, internal oblique and gluteus maximus $(P<0.05)$, but, not a significant difference in erector spinae and biceps femoris $(P>0.05)$ (Table 3).

In AQP, there were significant increases in Tlb, Tro, Pfe and Pro $(P<0.05)$, but not a significant difference in Tfe and $\mathrm{Plb}(P>$

Table 2. Changes of lumbopelvic motions and muscle activities in APB ( $n=8)$

\begin{tabular}{lccccl}
\hline \multicolumn{1}{l}{ Variable } & Pre & Post & Diff & $z$ & $P$-value \\
\hline \multicolumn{1}{l}{ Change of motion $\left(^{\circ}\right)$} & & & & \\
Tfe & $4.68 \pm 2.57$ & $1.72 \pm 1.09$ & $-2.96 \pm 2.76$ & -2.524 & $0.012^{*}$ \\
Tlb & $4.33 \pm 1.73$ & $0.95 \pm 0.57$ & $-3.38 \pm 1.47$ & -2.521 & $0.012^{*}$ \\
Tro & $2.73 \pm 2.47$ & $1.10 \pm 1.25$ & $-1.63 \pm 2.06$ & -1.820 & 0.069 \\
Pfe & $4.98 \pm 3.83$ & $0.92 \pm 0.61$ & $-4.06 \pm 3.83$ & -2.380 & $0.017^{*}$ \\
Plb & $0.95 \pm 0.57$ & $1.82 \pm 1.51$ & $-1.38 \pm 2.24$ & -1.540 & 0.123 \\
Pro & $7.73 \pm 1.71$ & $2.21 \pm 1.58$ & $-5.52 \pm 1.75$ & -2.521 & $0.012^{*}$ \\
Change of activity $(\% \mathrm{MVIC})$ & & & & \\
Rt. EO & $16.55 \pm 6.12$ & $25.19 \pm 12.11$ & $8.63 \pm 10.16$ & -2.240 & $0.025^{*}$ \\
Lt. EO & $13.32 \pm 10.44$ & $23.32 \pm 22.45$ & $9.99 \pm 12.72$ & -2.380 & $0.017^{*}$ \\
Rt. IO & $11.76 \pm 9.33$ & $28.92 \pm 16.12$ & $17.15 \pm 11.93$ & -2.380 & $0.017^{*}$ \\
Lt. IO & $25.64 \pm 12.63$ & $45.38 \pm 37.69$ & $19.74 \pm 17.06$ & -2.521 & $0.012^{*}$ \\
Rt. ES & $22.96 \pm 13.47$ & $28.22 \pm 19.35$ & $5.25 \pm 17.05$ & -0.700 & 0.484 \\
Lt. ES & $16.00 \pm 5.99$ & $16.86 \pm 10.48$ & $0.86 \pm 13.22$ & -0.280 & 0.779 \\
GM & $24.70 \pm 10.75$ & $58.23 \pm 17.16$ & $33.53 \pm 12.24$ & -2.521 & $0.012^{*}$ \\
BF & $38.26 \pm 14.65$ & $35.44 \pm 13.70$ & $-2.81 \pm 11.14$ & -0.560 & 0.575 \\
\hline
\end{tabular}

Values are presented as mean \pm standard deviation.

$\mathrm{APB}$, abdominal drawing-in maneuver with pressure biofeedback group; Tfe, thoracic flexion-extension; Tlb, thoracic lateral bending; Tro, thoracic rotation; Pfe, lumbopelvic flexion-extension; Plb, lumbopelvic lateral bending; Pro, lumbopelvic rotation; MVIC, maximal voluntary isometric contraction; Rt., right; Lt., left; EO, external oblique; IO, internal oblique; $\mathrm{ES}$, erector spinae; $\mathrm{GM}$, gluteus maximus; $\mathrm{BF}$, biceps femoris; Diff, difference value.

${ }^{*} P<0.05$.
Table 3. Changes of lumbopelvic motions and muscle activities in AFR ( $n=9)$

\begin{tabular}{lccccl}
\hline \multicolumn{1}{l}{ Variable } & Pre & Post & Diff & $z$ & $P$-value \\
\hline \multicolumn{2}{l}{ Change of motion $\left({ }^{\circ}\right)$} & & & & \\
Tfe & $3.96 \pm 2.64$ & $1.82 \pm 1.13$ & $-2.14 \pm 3.10$ & -1.605 & 0.108 \\
Tlb & $3.60 \pm 1.28$ & $1.31 \pm 1.98$ & $-2.29 \pm 2.06$ & -2.192 & $0.028^{*}$ \\
Tro & $3.90 \pm 2.63$ & $2.28 \pm 1.29$ & $-1.61 \pm 1.87$ & -2.314 & $0.021^{*}$ \\
Pfe & $6.35 \pm 2.90$ & $2.30 \pm 1.46$ & $-4.05 \pm 2.94$ & -2.521 & $0.012^{*}$ \\
Plb & $3.56 \pm 1.84$ & $1.88 \pm 1.66$ & $-1.67 \pm 1.82$ & -2.524 & $0.012^{*}$ \\
Pro & $10.07 \pm 3.27$ & $3.16 \pm 1.54$ & $-6.91 \pm 3.21$ & -2.666 & $0.008^{*}$ \\
Change of activity $(\% \mathrm{MVIC})$ & & & & \\
Rt. EO & $11.91 \pm 3.16$ & $25.76 \pm 13.82$ & $13.85 \pm 15.95$ & -2.429 & $0.015^{*}$ \\
Lt. EO & $10.36 \pm 4.57$ & $25.15 \pm 15.33$ & $14.79 \pm 15.83$ & -2.666 & $0.008^{*}$ \\
Rt. IO & $18.21 \pm 12.85$ & $66.18 \pm 26.37$ & $47.97 \pm 25.06$ & -2.666 & $0.008^{*}$ \\
Lt. IO & $42.14 \pm 22.23$ & $68.03 \pm 24.07$ & $25.89 \pm 24.32$ & -2.429 & $0.015^{*}$ \\
Rt. ES & $24.24 \pm 19.26$ & $30.13 \pm 24.10$ & $5.89 \pm 33.90$ & -0.415 & 0.678 \\
Lt. ES & $21.79 \pm 18.02$ & $37.90 \pm 23.29$ & $16.11 \pm 33.08$ & -1.599 & 0.110 \\
GM & $25.33 \pm 20.32$ & $57.78 \pm 17.06$ & $32.44 \pm 21.29$ & -2.666 & $0.008^{*}$ \\
BF & $36.85 \pm 13.89$ & $35.39 \pm 11.34$ & $-1.46 \pm 15.62$ & -0.178 & 0.859
\end{tabular}

Values are presented as mean \pm standard deviation.

AFR, abdominal drawing-in maneuver with foam-roller group; Tfe, thoracic flexion-extension; Tlb, thoracic lateral bending; Tro, thoracic rotation; Pfe, lumbopelvic flexion-extension; Plb, lumbopelvic lateral bending; Pro, lumbopelvic rotation; MVIC, maximal voluntary isometric contraction; Rt., right; Lt., left; EO, external oblique; 10 , internal oblique; ES, erector spinae; GM, gluteus maximus; BF, biceps femoris; Diff, difference value.

${ }^{*} P<0.05$.

Table 4. Changes of lumbopelvic motions and muscle activities in AQP ( $n=9)$

\begin{tabular}{lccccc}
\hline Variable & Pre & Post & Diff & $z$ & $P$-value \\
\hline \multicolumn{1}{c}{ Change of motion $\left(^{\circ}\right)$} & & & & \\
Tfe & $3.50 \pm 2.98$ & $1.10 \pm 0.52$ & $-2.40 \pm 3.18$ & -1.680 & 0.093 \\
Tlb & $4.32 \pm 2.73$ & $1.18 \pm 1.33$ & $-3.13 \pm 2.66$ & -2.240 & $0.025^{*}$ \\
Tro & $2.63 \pm 1.64$ & $0.93 \pm 0.86$ & $-1.67 \pm 1.66$ & -2.028 & $0.043^{*}$ \\
Pfe & $5.01 \pm 2.13$ & $2.18 \pm 1.13$ & $-2.82 \pm 1.97$ & -2.366 & $0.018^{*}$ \\
Plb & $3.88 \pm 3.10$ & $2.40 \pm 1.96$ & $-1.48 \pm 2.57$ & -1.682 & 0.092 \\
Pro & $8.51 \pm 3.08$ & $3.08 \pm 0.95$ & $-5.42 \pm 3.08$ & -2.521 & $0.012^{*}$ \\
Change of activity $(\% \mathrm{MVIC})$ & & & & \\
Rt. EO & $16.37 \pm 6.23$ & $29.27 \pm 15.00$ & $12.89 \pm 12.63$ & -2.240 & $0.025^{*}$ \\
Lt. EO & $15.64 \pm 19.31$ & $26.19 \pm 27.77$ & $10.55 \pm 9.41$ & -2.521 & $0.012^{*}$ \\
Rt. IO & $26.74 \pm 23.15$ & $37.41 \pm 26.08$ & $10.66 \pm 19.42$ & -1.400 & 0.161 \\
Lt. IO & $48.58 \pm 31.98$ & $86.00 \pm 11.58$ & $37.41 \pm 26.79$ & -2.521 & $0.012^{*}$ \\
Rt. ES & $17.39 \pm 8.53$ & $22.48 \pm 11.55$ & $5.08 \pm 9.03$ & -1.400 & 0.161 \\
Lt. ES & $24.61 \pm 15.87$ & $35.15 \pm 27.6$ & $10.53 \pm 24.35$ & -1.400 & 0.161 \\
GM & $20.31 \pm 8.47$ & $46.15 \pm 16.69$ & $25.84 \pm 13.66$ & -2.521 & $0.012^{*}$ \\
BF & $32.15 \pm 13.80$ & $28.03 \pm 13.79$ & $-4.11 \pm 13.98$ & -0.700 & 0.484 \\
\hline
\end{tabular}

Values are presented as mean \pm standard deviation.

$A Q P$, abdominal drawing-in maneuver with quadruped group; Tfe, thoracic flexion-extension; Tlb, thoracic lateral bending; Tro, thoracic rotation; Pfe, lumbopelvic flexion-extension; $\mathrm{Plb}$, lumbopelvic lateral bending; Pro, lumbopelvic rotation; MVIC, maximal voluntary isometric contraction; Rt., right; Lt., left; EO, external oblique; IO, internal oblique; ES, erector spinae; GM, gluteus maximus; BF, biceps femoris; Diff, difference value.

${ }^{*} P<0.05$. 
0.05). There were significant increases in external oblique, Left internal oblique and gluteus maximus $(P<0.05)$, but not a significant difference in right internal oblique, erector spinae and biceps femoris $(P>0.05)$ (Table 4).

In the comparison of three groups, right internal oblique muscle activity alone showed a significant difference $(P<0.05)$. And there were significant differences between the pressure biofeedback group and foam-roller group, and between the pressure foam-roller group and quadruped group $(P<0.017)$.

\section{DISCUSSION}

The purpose of this study was to investigate the effects of the ADIM with three different methods on lumbopelvic motions, especially on rotation, and muscle activities in lumbar rotation syndrome. Findings of the present study showed significant decreases in the lumbopelvic rotation degree in all the groups, with lumbopelvic rotation degree becoming near to the normal range after interventions. It is considered that the recovery was due to ADIM which was applied to all groups. ADIM is effective to activates deep muscles of trunk, such as transvers abdominis, minimizes superficial muscles activities, and provides trunk stability (Hodges and Richardson, 1999). When extremities start moving, transvers abdominis is the first recruited muscle for stabilized posture and contributes to lumbar stability (Kim and Kim, 2015). If transvers abdominis contracts late, the lumbopelvic dysfunction could be caused (Hodges and Bui, 1996). When transvers abdominis, internal oblique, and external oblique contracted at the same time, they increased abdominal pressure and gave support to the spinal stability (McGill et al., 2003). It is considered that the increased power of transvers abdominis and internal oblique might decrease lumbopelvic rotation degree and contribute to trunk stability.

All groups showed significant increases in both external oblique activities. External oblique limits lumbar rotation by restricting lumbopelvic rotation, and when lumbopelvic region moves external oblique contributes to stability of the lumbar segments (Kim et al., 2017; Sahrmann et al., 2017). In addition, a study reported that trunk rotation caused increased muscle activities of transvers abdominis, external oblique, and internal oblique (Cresswell et al., 1992; Ferreira et al., 2004; Lee et al., 2013). Therefore, it is possible to say that all ADIM exercises caused the increases of external oblique activities during single hip extension; consequently, they were effective to reduce lumbopelvic rotation degree by increasing lumbopelvic stability.

For the change of internal oblique activity, except right internal oblique of AQP, left internal oblique of AQP and internal oblique of both sides of other two groups showed significant increases. Internal oblique not only limits trunk rotation but is also the most effective muscle while hip flexion (Kim et al., 2017). A previous study reported that internal oblique influenced the lumbopelvic neutral position, spinal posture, and equilibrium between inside and outside of trunk (Callaghan et al., 1998). Therefore, it is possible to say that left hip flexion posture for supporting the balance of body increased the muscle activity of left internal oblique in all groups commonly during single hip extension in quadruped position. Moreover, it is considered that in responsible to lumbopelvic rotation toward left side by left internal oblique, right internal oblique contracted to contribute equilibrium to the body; consequently, the muscle activity of right internal oblique also increased. That is, when a lower extremity started moving, transverse abdominis, which was contracted in advance, contributed to stability of the lumbar spine, and for the left hip flexion supporting the body on the floor, left internal oblique was contracted and caused contraction of right internal oblique.

All groups showed significant increases on the activity of gluteus maximus. Gluteus maximus causes the hip extension and hip external rotation, while it would be weakened lumbopelvic region postures swayback and is activated minimally during stance phase (Kim and Kim, 2015). In this study, it is considered that the increased activity of gluteus maximus might be caused by the effectiveness of ADIM exercises performed to achieve stability on lumbopelvic region. This study showed significant decreases on Tfe in all groups. A previous study about the difference of the muscle activity between with and without ADIM during prone hip extension reported that ADIM group showed a significant increases of the activity of gluteus maximus and significant decrease of the lumbopelvic flexion degree (Oh et al., 2007).

Right internal oblique showed significant differences between APB and AFR, and between AFR and AQP. When subjects performed the right single hip extension after each intervention, they showed greater muscle activities on left internal oblique and lesser degrees of lumbopelvic rotation than before. Thus, it is considered that right internal oblique was activated more strongly to keep balance during right single hip extension.

In the comparison of the three groups, there was not a significant difference in lumbopelvic rotation degree. However, lumbopelvic rotation degree was reduced in all groups. A previous study compared the effects of single leg flexion between with hook-lying posture on a flat surface and with a foam-roller, and reported that the latter caused more abdominal muscle activity 
than the former (Kim et al., 2011). In addition, another study compared the effects of prone knee extension between on a floor and on a foam-roller, and reported the movement on the foam-roller improved trunk stability more than the movement on the floor (Kim et al., 2013a).

In summary, although the changes of muscle activities appeared differently in each group, lumbopelvic rotation degree was significantly decreased in all groups. Especially, AFR showed the most significant improvement on lumbopelvic rotation. ADIM exercises with the three different methods were all effective to reduce the lumbopelvic rotation degree. Therefore, researchers and clinicians should be able to differentiate and apply the proper exercise method regarding the conditions of patients including muscle power, balancing ability, and the muscle activities that work for the lumbopelvic stability and normal movement.

The current study has some limitations. All subjects had the lumbopelvic rotation symptom but they did not have pain. Further studies should be done with the patients who have pain, and the results compared to those of this study. The number of subjects was small, and it is difficult to generalize the results, so further studies should be done to confirm the present findings. In the analysis of the activity of internal oblique, right internal oblique of AQP alone did not show significant difference. This issue should be explicated by means if in-depth research. In addition, this study was cross-sectional, so longitudinal follow-up should be done to determine the long-term effects of the three different ADIM exercises for patients with lumbar rotation syndrome.

\section{CONFLICT OF INTEREST}

No potential conflict of interest relevant to this article was reported.

\section{REFERENCES}

Callaghan JP, Gunning JL, McGill SM. The relationship between lumbar spine load and muscle activity during extensor exercises. Phys Ther 1998;78:8-18.

Cresswell AG, Grundström H, Thorstensson A. Observations on intra-abdominal pressure and patterns of abdominal intra-muscular activity in man. Acta Physiol Scand 1992;144:409-418.

Cynn HS, Oh JS, Kwon OY, Yi CH. Effects of lumbar stabilization using a pressure biofeedback unit on muscle activity and lateral pelvic tilt during hip abduction in sidelying. Arch Phys Med Rehabil 2006;87:
1454-1458.

Ferreira PH, Ferreira ML, Hodges PW. Changes in recruitment of the abdominal muscles in people with low back pain: ultrasound measurement of muscle activity. Spine (Phila Pa 1976) 2004;29:2560-2566.

Hall L, Tsao H, MacDonald D, Coppieters M, Hodges PW. Immediate effects of co-contraction training on motor control of the trunk muscles in people with recurrent low back pain. J Electromyogr Kinesiol 2009; 19:763-773.

Hodges PW, Bui BH. A comparison of computer-based methods for the determination of onset of muscle contraction using electromyography. Electroencephalogr Clin Neurophysiol 1996;101:511-519.

Hodges PW, Richardson CA. Altered trunk muscle recruitment in people with low back pain with upper limb movement at different speeds. Arch Phys Med Rehabil 1999;80:1005-1012.

Kim JS, Seok CH, Jeon HS. Abdominal draw-in maneuver combined with simulated weight bearing increases transversus abdominis and internal oblique thickness. Physiother Theory Pract 2017;33:954-958.

Kim JW, Han JY, Kang MH, Ha SM, Oh JS. Comparison of posterior oblique sling activity during hip extension in the prone position on the floor and on a round foam roll. J Phys Ther Sci 2013a;25:977-979.

Kim SJ, Kwon OY, Yi CH, Jeon HS, Oh JS, Cynn HS, Weon JH. Comparison of abdominal muscle activity during a single-legged hold in the hook-lying position on the floor and on a round foam roll. J Athl Train 2011;46:403-408.

Kim TW, Kim YW. Effects of abdominal drawing-in during prone hip extension on the muscle activities of the hamstring, gluteus maximus, and lumbar erector spinae in subjects with lumbar hyperlordosis. J Phys Ther Sci 2015;27:383-386.

Kim YR, Kim JW, An DH, Yoo WG, Oh JS. Effects of a pelvic belt on the EMG activity of the abdominal muscles during a single-leg hold in the hook-lying position on a round foam roll. J Phys Ther Sci 2013b; 25:793-795

Lee AY, Kim EH, Cho YW, Kwon SO, Son SM, Ahn SH. Effects of abdominal hollowing during stair climbing on the activations of local trunk stabilizing muscles: a cross-sectional study. Ann Rehabil Med 2013; 37:804-813.

Matthijs OC, Dedrick GS, James CR, Brismée JM, Hooper TL, McGalliard MK, Sizer PS Jr. Co-contractive activation of the superficial multifidus during volitional preemptive abdominal contraction. PM R 2014;6: 13-21.

McGill SM, Grenier S, Kavcic N, Cholewicki J. Coordination of muscle activity to assure stability of the lumbar spine. J Electromyogr Kinesiol 2003;13:353-359.

Oh JS, Cynn HS, Won JH, Kwon OY, Yi CH. Effects of performing an abdominal drawing-in maneuver during prone hip extension exercises 
on hip and back extensor muscle activity and amount of anterior pelvic tilt. J Orthop Sports Phys Ther 2007;37:320-324.

Pirouzi S, Emami F, Taghizadeh S, Ghanbari A. Is Abdominal muscle activity different from lumbar muscle activity during four-point kneeling? Iran J Med Sci 2013;38:327-333.

Sahrmann S, Azevedo DC, Dillen LV. Diagnosis and treatment of movement system impairment syndromes. Braz J Phys Ther 2017;21:391-399.

Scholtes SA, Gombatto SP, Van Dillen LR. Differences in lumbopelvic motion between people with and people without low back pain during two lower limb movement tests. Clin Biomech (Bristol, Avon) 2009;24: 7-12.

Van Deun S, Staes FF, Stappaerts KH, Janssens L, Levin O, Peers KK. Relationship of chronic ankle instability to muscle activation patterns during the transition from double-leg to single-leg stance. Am J Sports Med 2007;35:274-281.

Weyrauch SA, Bohall SC, Sorensen CJ, Van Dillen LR. Association between rotation-related impairments and activity type in people with and without low back pain. Arch Phys Med Rehabil 2015;96:1506-1517. 